\title{
A Novel Enzyme-Triggered Domino Reaction Producing Fluorescent Quinoxalin-2(1H)-one-based Heterocycles
}

\author{
Garance Dejouy, ${ }^{\dagger}, \dot{\dagger}$ Kévin Renault, ${ }^{*}, \dagger+{ }^{\dagger}$ Quentin Bonnin, ${ }^{\dagger}$ Arnaud Chevalier, ${ }^{\Uparrow}$ Cédric Michaudet, ${ }^{\dagger}$ Michel \\ Picquet, ${ }^{\dagger}$ Ibai E. Valverde, ${ }^{\dagger}$ and Anthony Romieu ${ }^{*} \dagger$
}

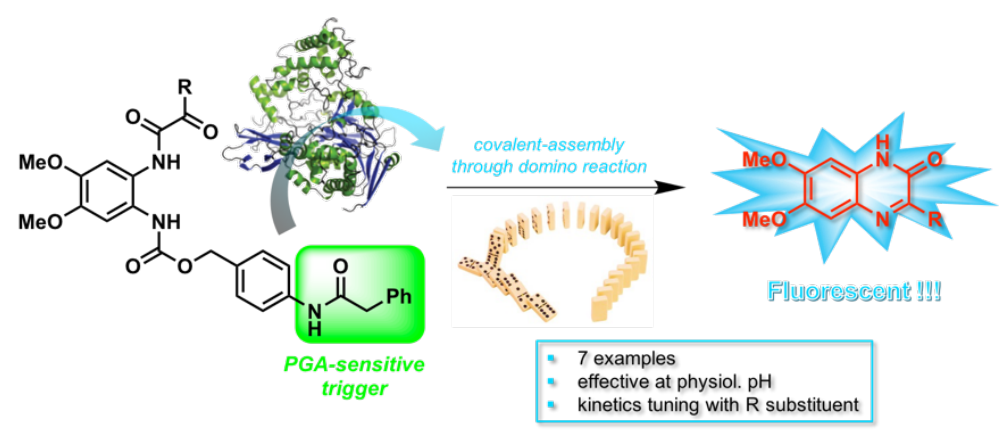

A simple and effective biocompatible domino reaction triggered by a model protease and leading to formation of strongly fluorescent quinoxalin-2(1H)-one $N$-heterocycles is described. Some positive attributes including versatility and ability to provide outstanding fluorescence "OFF-ON" responses were revealed by this work. They open the way for practical applications of this novel type of "covalent-assembly" based fluorescent probes in the fields of sensing and bioimaging.

Among the myriad of synthetic transformations and catalysts currently available for concise and efficient synthesis of organic molecules, bioinspired approaches based on the use of enzymes often intertwined with cascade/domino processes, occupy a special place and are receiving greater attention of late. ${ }^{1}$ They have also contributed to the emergence of in vivo chemistry (often named as intracellular chemistry $)^{2}$ that can be defined as the use of living cells as reaction vessels and their constituents (e.g., enzymes) possibly together with abiotic metal catalysts, ${ }^{3}$ to achieve one or several reactions for internal construction of sophisticated nano-objects from exogenous synthetic precursors. In addition to possible applications devoted to the production of high-value chemicals, one of the most striking achievements in this emerging area is undoubtedly the biocompatible "click" reaction between an aromatic nitrile (e.g., 6-amino-2-cyanobenzothiazole (CBT)) and D-cysteine leading to D-luciferin scaffold, ${ }^{4}$ discovered by the Rao group in 2010 (Figure 1, top). ${ }^{5}$ Its ability to be effective for the controlled assembly of nanoparticles in living cells together with its possible triggering by a biostimulus ( $\mathrm{pH}$, redox status and/or hydrolytic enzymes) has fostered the emergence of cutting-edge reactivity-based sensing approaches for in vivo molecular imaging, especially through the bioluminescence modality, for addressing challenges associated to light-based diagnosis. ${ }^{6}$

These remarkable achievements perfectly illustrate both utility and benefits of biocompatible/bioorthogonal transformations that are capable of generating a bright luminescence output signal upon the action of a biological trigger. In the field of activity-based fluorescent sensing, a new probe design principle namely the "covalent-assembly" approach, pioneered by the Swager group with the design of a "smart" semiconductive polymer for fluorogenic detection of fluoride ions, ${ }^{7}$ and next rationalized by Anslyn and Yang to address relevant biological questions, ${ }^{8,9}$ has emerged in the mid-2000s. This cutting-edge strategy is based on in situ formation of a fluorophore from a caged compound (not belonging to major classes of fluorescent dyes and, in theory at least, devoid of light emission ability) through a domino reaction triggered by the species to be detected and ideally designed to work properly in aqueous media (Figure 1, middle and bottom). Since this novel class of activatable fluorescent probes are supposed to dramatically improve signal-to-noise ratio $(\mathrm{S} / \mathrm{N})$ responses, thus providing optimal detection sensitivity, it is not really surprising that they have rapidly become popular tools for specific detection and imaging of enzyme activities (mainly, hydrolases and reductases). The vast majority of "covalent-assembly" based fluorescent probes devoted to enzyme biosensing involve in situ formation of blue-green emitting (2-imino)coumarins or related fluorophores through analyte-triggered lactonization or Pinner cyclization reactions ${ }^{10,11,12}$ even if some of these published examples are also regarded as fluorogenic probes undergoing rigidification of their floppy pre-existing push-pull system upon the action of the bioanalyte. ${ }^{8 \mathrm{~b}}$ Our group, inspired by work of Yang et $a l .,{ }^{13}$ have recently contributed to this research field by expanding the scope to longer-wavelength fluorophores belonging to the popular class of xanthene dyes (i.e., unsymmetrical pyronins with fluorescence features within the yellow-orange spectral region). ${ }^{14}$ Some notable results were obtained with 
several hydrolytic enzymes including penicillin $G$ acylase (PGA) and leucine amino peptidase (LAP) and we demonstrated that a subtle equilibrium between reactivity and stability of caged precursors of pyronins can be readily obtained by means of effective and facile structural modifications.
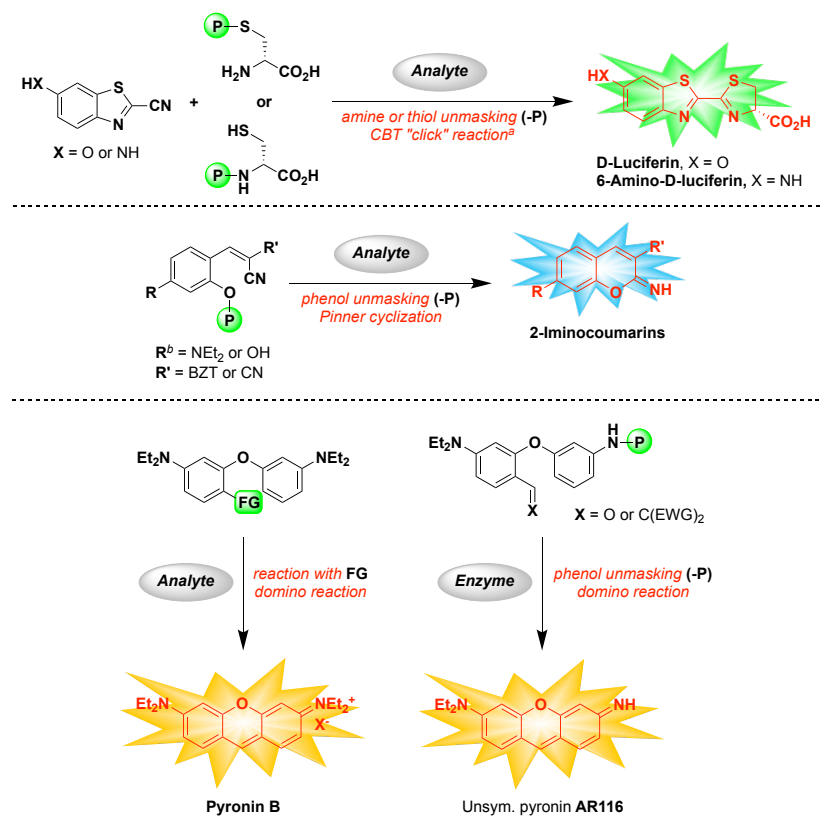

Figure 1. Background information about in situ synthesis of bioluminophores (top) and fluorophores (2-iminocoumarins (middle) and pyronins (bottom)) triggered by the (bio)analyte to be detected. (BZT $=2$-benzothiazolyl, EWG = electron-withdrawing group, $\mathrm{FG}=$ functional group that reacts with the targeted analyte, $\mathrm{P}=$ recognition moiety of the targeted enzyme). ${ }^{a}$ For clarity, related polymerization reactions and intramolecular cyclizations leading to nano-aggregates were omitted. ${ }^{b}$ Please note: fused julolidine, 4-dimethylaminophenyl and 1,4-diethylpiperazine fragments are also frequently used in the design of such "caged" precursors, to red-shift spectral features of in situ formed 2-iminocoumarin.

Despite all these advances, "covalent-assembly" fluorogenic probes may sometimes have some drawbacks including (1) intrinsic red or far-red fluorescence due to aggregation-induced emission (AIE) phenomenon that prevents intensometric "OFFON" detection, ${ }^{14 b, 15}$ and (2) limited molecular diversity that may complicate optimization of their properties for a given application. Thus, there is a clear need to identify alternative fluorescent scaffolds that can be built by the targeted enzyme through a novel domino process. We have identified the quinoxalin-2(1H)-ones, a popular class of benzo-fused $N$-heterocycles with a wide spectrum of pharmacological activities. ${ }^{16}$ They are frequently used as skeletons for the design of biologically active compounds and their spectral behavior close to those of 7-(dialkylamino)/7-hydroxycoumarins has recently promoted their use either as fluorophore alone or as optical reporter in "smart" fluorescent probes acting as chemodosimeters or chemosensors. ${ }^{17}$ Furthermore, the very old condensation reaction historically used to prepare these $N$-heterocycles is easy to implement and involves the use of ortho-phenylenediamine and $\alpha$-ketoacid (or $\alpha$-ketoester derivative) as coupling partners. ${ }^{18}$ Its versatility stems from the commercial or synthetic availability of a wide range of these starting materials bearing various substituents. We hypothesized that the cyclization/aromatization process which completes the $N$-heterocycle construction may occur spontaneously under physiological conditions. In the light of the mechanism generally accepted for this reaction, i.e., the ketone moiety undergoing an intramolecular nucleophilic addition of the neighboring primary amino group followed by loss of a water molecule, it is reasonable to assume that a caged orthophenylenediamine derivative, whose the primary amino group would be acylated with an enzyme-specific trigger recognition moiety (the second one being acylated with the selected $\alpha$-keto acid), should act as an effective "covalent-assembly" based fluorescent probe (Figure 2).

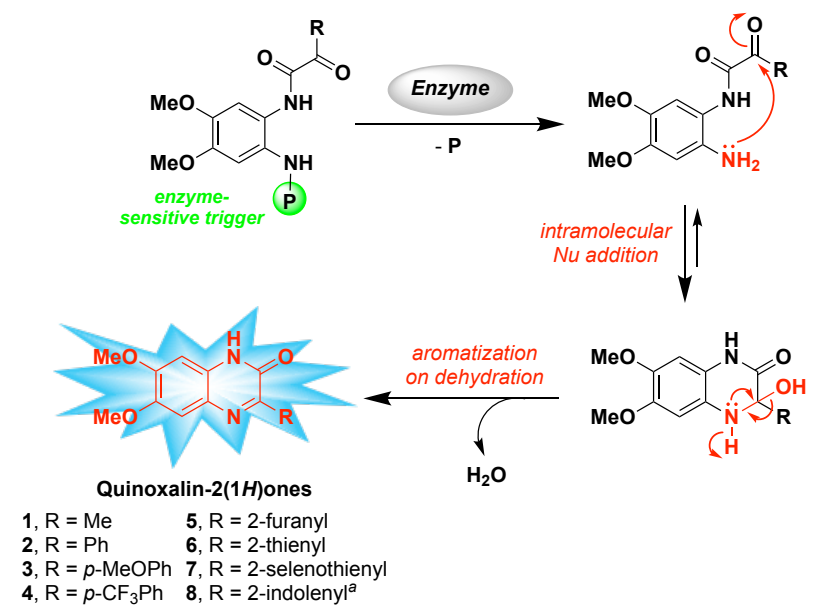

Figure 2. Enzyme sensing strategy explored in this work, based on the use of "covalent-assembly" fluorogenic probes whose activation leads to in situ formation of quinoxalin-2(1H)-ones. ${ }^{a}$ Due to its poor stability, we failed to synthesize the corresponding "covalent-assembly" fluorescent probe.

We report here the practical implementation of this unusual fluorogenic reaction-based process through the synthesis of seven distinct $N$-caged ortho-phenylenediamine derivatives and using PGA as a model protease, a commercially available and widely used biocatalyst in the enzymatic synthesis of $\beta$-lactam antibiotics through the hydrolysis of phenylacetamide moieties. ${ }^{19}$ The ability of this enzyme to trigger in situ formation of fluorescent quinoxalin-2(1H)-ones from these "covalent-assembly" type probes was studied in detail through in vitro fluorescence assays and HPLC-fluorescence/-MS analyses. All data generated have been used to rationalize the reaction mechanism and the influence of alkyl/aryl substituent of $\alpha$-ketoamide moiety on the overall efficiency of this novel domino process.

The planned synthetic strategy was based on the sequential $\mathrm{N}$-acylation of 4,5-dimethoxy-1,2-phenylenediamine 9 with para-nitrophenyl carbonate derivative of para-(phenylacetamido)benzyl alcohol (PhAc-PABA) $\mathbf{1 0}^{20}$ and $\alpha$-ketoacid chloride to install the recognition unit of PGA and electrophilic moiety respectively (Scheme 1). The substitution pattern of 2-aminoaniline 9 was chosen because the quinoxalin-2(1H)-ones obtained from this starting synthon are known to be the most fluorescent candidates form this class of benzo-fused $N$-heterocycles. Since 9 was found to be partially unstable over prolonged storage (degradation was observed after less than 2 months at $-20{ }^{\circ} \mathrm{C}$ and under argon atmosphere), it was prepared immediately prior to use by hydrogenation over $\mathrm{Pd} / \mathrm{C}$ catalyst of commercial, stable 4,5-dinitroveratrole. ${ }^{21}$ The phenylacetamide unit known to be recognized and cleaved by PGA, was connected to 1,2phenylenediamine scaffold through a self-immolative linker (i.e., para-hydroxybenzyl) to lower steric hindrance and hence increase accessibility of the probe to the enzyme's active site. ${ }^{22}$ Indeed, our preliminary investigations have shown that direct $N$-acylation of 9 with phenylacetyl chloride led to unreactive "covalent-assembly" fluorogenic probes, not recognized by PGA (data not shown). Molecular diversity was readily 
achieved by selecting 7 different $\alpha$-ketoacids including pyruvic acid or derivatives arising formally from benzylic oxidation of phenylacetic or heteroarylacetic acid (phenyl, para-methoxyphenyl, para-(trifluoromethyl)phenyl, 2-furyl, 2-thienyl or 2selenothienyl as fragment). They are commercially available or easily prepared by $\mathrm{SeO}_{2}$-mediated oxidation of the corresponding $\alpha$-methylketone (for details, see the Supporting Information). Conversion into the corresponding acyl chlorides was achieved by treatment with an excess of dichloromethyl methyl ether (acting as both chlorinating agent and solvent) at $50{ }^{\circ} \mathrm{C}$, except for 2-selenopheneglyoxylic acid found to be unstable under these conditions; $\mathrm{SOCl}_{2}$ in dry DMF was used in this latter case. Practical implementation of the two distinct $N$-acylation reactions was performed as follows: first, 1,2-phenylenediamine 9 was reacted with activated carbonate $\mathbf{1 0}$ (stoichiometrically deficient relative to $9,0.36$ equiv.) in dry DMF, in the presence of 1-hydroxybenzotriazole (HOBt, 0.36 equiv.) and TEA (1 equiv.). Thereafter, amidation of the remaining primary aniline with $\alpha$-ketoacid chloride (1.2 equiv.) was conducted under nearly identical conditions (in dry DMF, in the presence of TEA). Purification by flash-column chromatography over silica gel provided the PGA-responsive fluorogenic probes in moderate to good yields (23-69\%). All spectroscopic data (see the Supporting Information), especially multinuclear NMR and high- and low-resolution mass spectrometry), were in agreement with the structures assigned. Interestingly, selenophenebased probe 18 was further characterized by ${ }^{77} \mathrm{Se}$ NMR (proton decoupled experiment) and the single resonance peak at 675.3 ppm is a valuable evidence for the presence of a single species within the product sample (Figure S114).

Scheme 1. Synthesis of PGA-responsive "covalent-assembly" fluorogenic probes 12-18

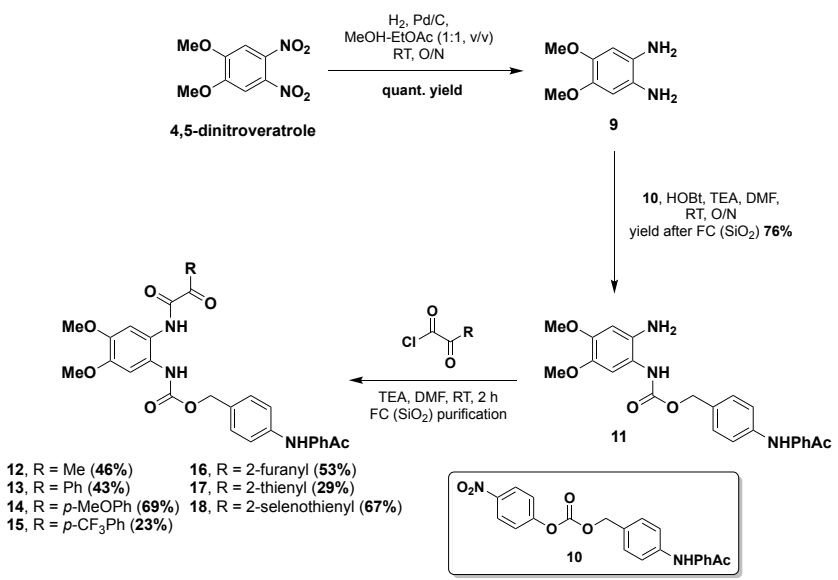

We have also prepared the quinoxalin-2(1H)-one fluorophores 1-7 assumed to be formed upon the enzymatic activation of probes 12-18. Indeed, their use as references in fluorescence assays and HPLC analyses is essential to clearly demonstrate the reaction-based sensing mechanism (see the Supporting Information for synthesis and characterization of fluorophores 17). The synthesis of indole-based derivative $\mathbf{8}$ was also achieved but we failed to obtain the corresponding PGA-responsive probe owing to its poor stability. To have an indication of the magnitude of fluorogenic "OFF-ON" response arising from PGA activation of probes 12-18, photophysical properties of fluorophores were also determined both in phosphate buffer (PB, $100 \mathrm{mM}, \mathrm{pH}$ 7.6) and DMSO (see Tables S1 and S2, and Figures S115-S130). Compounds $\mathbf{1 - 3}$ and $\mathbf{5}$ have been identified as the most brilliant dyes within the violet-blue-green spectral range typical for quinoxalin-2(1H)-ones $\left(B\left(\varepsilon \times \Phi_{\mathrm{F}}\right)\right.$ values within the range $5000-9100 \mathrm{M}^{-1} \mathrm{~cm}^{-1}$ for PB medium). It is worth noting that fluorescence quantum yield decreases from 0.37 for furane-based dye $\mathbf{5}$ to 0.20 for thiophene-based dye 6 to 0.06 for selenophene-based dye 7 which is consistent with heavy-atom effects promoting triplet formation relative to fluorescence. ${ }^{23}$ Another interesting feature that will be subjected to additional investigations, concerns the indole-based dye 8 which was found to be almost non-fluorescent $\left(\Phi_{\mathrm{F}}<0.01\right)$ in $\mathrm{PB}$, probably as a consequence of photoinduced electron transfer $(\mathrm{PeT})$ process. ${ }^{24}$ Conversely, preliminary fluorescence fixed-cell imaging experiments have shown the emission ability of this quinoxalin-2(1H)-one in a real biological context and its superior capability for cellular staining compared to the phenylbased dye $\mathbf{2}$ otherwise characterized by a dramatically higher $\Phi_{\mathrm{F}}$ in PB (0.6) (Figures S66-S67). This campaign of photophysical studies was completed by spectral measurements that confirmed the lack of fluorescence emission in PB for all PGAresponsive "covalent-assembly" type probes 12-18.

Fluorogenic PGA assays and blank experiments (that confirmed hydrolytic stability of compounds 12-18) were achieved through time-course measurements following a reliable protocol previously used by us. ${ }^{11 f, 14}$ In all cases, addition of recombinant PGA (from Escherichia coli) caused a rapid increase of blue fluorescence emission centered at a wavelength value within the range $455-490 \mathrm{~nm}$, that reached a plateau in less than 10 min (Figure 3 and Figures S138-S151), except for the probe 15 bearing para-(trifluoromethyl)phenyl moiety as the ketone substituent. Electron-withdrawing effect of $-\mathrm{CF}_{3}$ may negatively impact the last step of domino process namely dehydration, leading to quinoxalin-2(1H)-one aromatization (Figure 2); this rate-determining step should be favored by combined push electron effects of $N$ atom and neighboring alkyl/aryl substituent toward carbon atom center undergoing the loss of hydroxyl group.

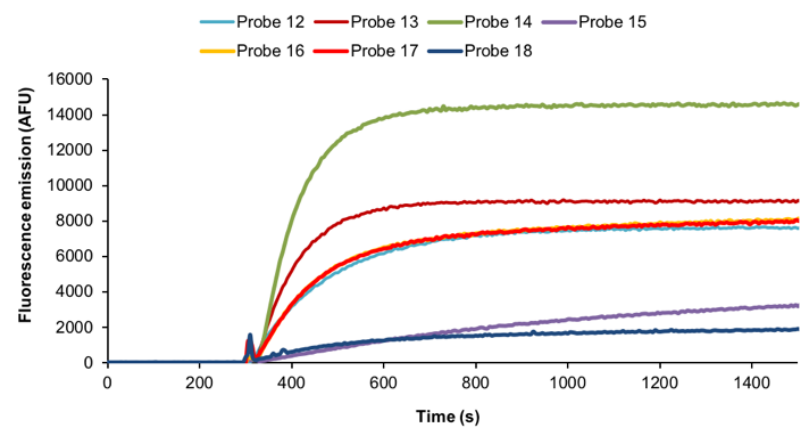

Figure 3. Time-dependent changes in the blue-green fluorescence intensity (Ex./Em. at the respective absorption/emission maxima, distinctive for each fluorophore, see the Supporting Information, slit $=2 \mathrm{~nm}$ ) of fluorogenic probes 12-18 (concentration: 1.0 $\mu \mathrm{M})$ in the presence of PGA (1 U) in PB ( $100 \mathrm{mM}$, pH 7.6) at $37^{\circ} \mathrm{C}$. Please note: PGA was added after $5 \mathrm{~min}$ of incubation of probe in $\mathrm{PB}$ alone.

Outstanding fluorescence "OFF-ON" responses were obtained even for the lowest fluorescent derivative bearing 2-selenothienyl moiety (Figures S139, S141, S143, S145, S147, S149 and S151). This highlights the main valuable feature of "covalent-assembly" approach in a striking manner. Furthermore, enzymatic activation and fluorogenic response arising from this was not impacted by the presence of biological interferents such as glutathione (Figures S152-S158). To confirm that the intense blue fluorescence signal detected was due to in situ formation of quinoxalin-2(1H)-one $N$-heterocycle, each 
enzymatic reaction mixture was subjected to RP-HPLCfluorescence analyses (Figure 4 for phenyl derivative $\mathbf{1 3}$ and Figures S159-S207). For each sample, a single peak was detected and unambiguously assigned to the expected fluorophore (comparison of retention times and co-injection with authentic samples of quinoxalin-2(1H)-ones 1-7 independently prepared, vide supra). To prove the validity of our assumption that PGAinitiated domino reaction to yield these benzo-fused $N$-heterocycles, the same enzymatic mixtures were next analyzed by RPHPLC-MS both in "full-scan" and single ion monitoring (SIM) modes (Figure 5 for phenyl derivative $\mathbf{1 3}$ and Figures S208$\mathrm{S} 235)$. The disappearance of the probe 13 peak $\left(t_{\mathrm{R}}=5.0 \mathrm{~min}\right)$ and unambiguous quantitative formation of quinoxalin-2(1H)one $2\left(t_{\mathrm{R}}=4.1 \mathrm{~min}\right)$ were clearly observed and both structure and integrity of fluorophore were supported by MS-ESI $+/$ - and UV-vis data. Interestingly no trace of elusive intermediate aniline arising from PGA-mediated removal of PhAc moiety was detected, confirming the high reactivity of its primary amino group toward the adjacent carbonyl of $\alpha$-ketoamide fragment. It should also be noted that both the existence and presence in enzymatic mixtures of the transient 2-hydroxy-1,2-dihydroquinoxalinone-type intermediate (i.e., intermediate undergoing dehydration, Figure 2) cannot be confirmed due to its rapid conversion to final fluorophore under acidic conditions of mobile phases used for RP-HPLC-MS analyses. Nevertheless, all results presented here allowed us to confirm both viability and effectiveness of the sensing mechanism initially claimed and depicted in Figure 2. Furthermore, depending on the electronic effects of $\mathrm{R}$ substituent found in the probe's structure, the reactivity of $\alpha$-ketoamide fragment and by extension the quinoxalinone-production rate, are impacted. This paves the way to a trivial molecular design strategy for fine-tuning reactivity of these quinoxalinone caged precursors.

In summary, we managed to expand the scope of "covalentassembly" fluorescent probe design principle to quinoxalin$2(1 H)$-ones. Indeed, the facile construction of such benzo-fused $\mathrm{N}$-heterocycles from structurally simple caged precursors and through an effective cyclization/dehydration sequence triggered by a model protease was demonstrated. We have also shown that kinetics of this fluorogenic process is dramatically influenced by the substituent nature of $\alpha$-ketoamide fragment (found in C-3 position of quinoxalin- $2(1 \mathrm{H})$-one formed in situ); the latter also having a significant impact on fluorescence properties and hence on the quality of detection. This feature combined to the wide scope of this approach (i.e., facile exchange of the recognition moiety introduced on the primary aniline of probe for targeting a wide range of distinct bioanalytes), will open new perspectives in the field of activity-based optical sensing/imaging and, more broadly, expand the molecular toolbox for in vivo chemsitry. Lastly, the presented enzyme-triggered cyclizaion/aromatization reaction could be a useful source of inspiration to medicinal/or chemists to devise novel advanced prodrug strategies. ${ }^{25}$
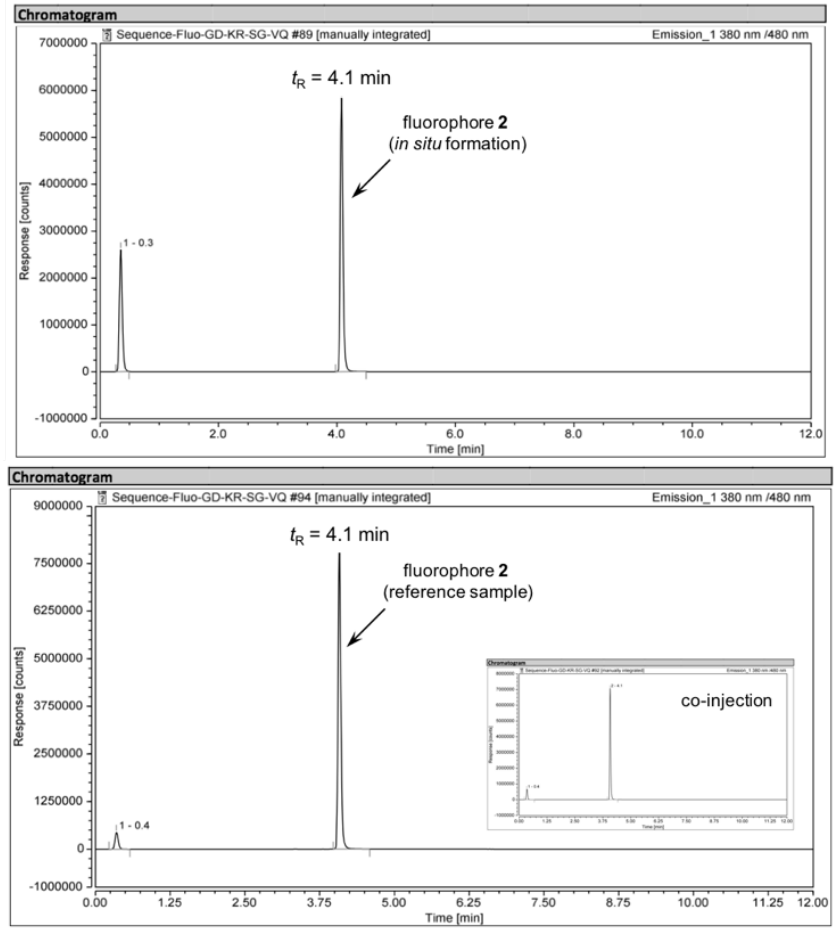

Figure 4. RP-HPLC elution profiles (fluorescence detection Ex./Em. 380/480 nm, see the Supplementary Information for elution conditions) of enzymatic reaction mixture of phenyl-based probe 13 with PGA (top) and authentic sample of fluorophore $\mathbf{2}$ (bottom) and co-injection of both (inset).
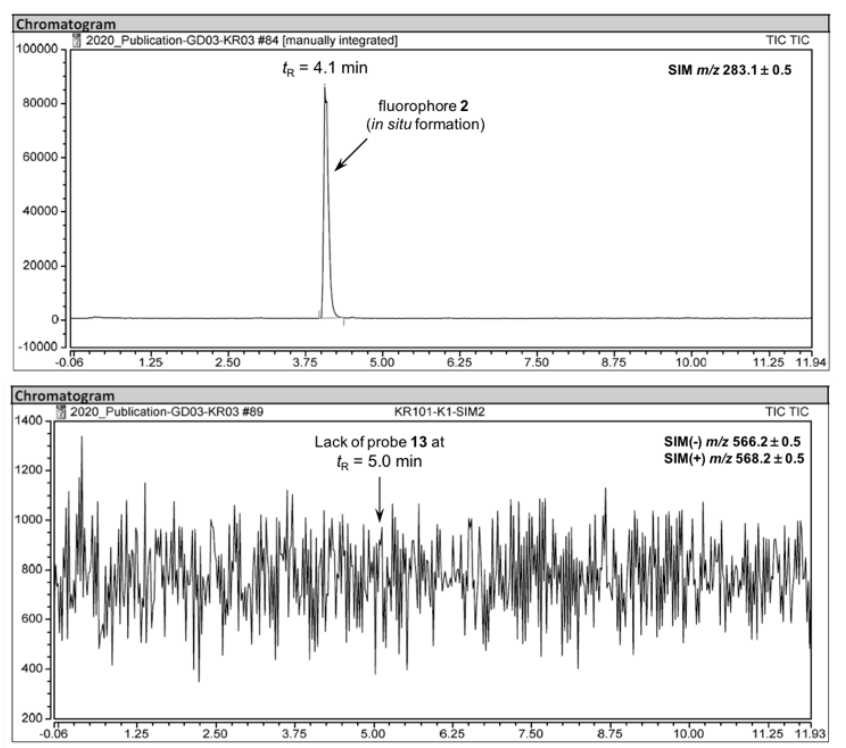

Figure 5. RP-HPLC elution profile (ESI-MS detection, SIM mode: ESI+ for detecting fluorophore (top) and ESI-/+ probe (bottom)) of enzymatic reaction mixture of phenylbased probe 13 with PGA.

\section{ASSOCIATED CONTENT}

\section{Supporting Information}

Experimental procedures, spectroscopic and analytical data, IR, NMR, MS and UV-vis/fluorescence spectra, elution profiles for all RP-HPLC-fluorescence/-MS analyses (PDF). The Supporting Information is available free of charge at xxx. 


\section{AUTHOR INFORMATION}

\section{Corresponding Authors}

Kévin Renault - Institut de Chimie Moléculaire de l'Université de Bourgogne, UMR 6302, CNRS, Univ. Bourgogne Franche-Comté, 9, Avenue Alain Savary, 21000, Dijon, France; 0000-0003-44278146; E-mail: kevin.renault@u-bourgogne.fr

Anthony Romieu - Institut de Chimie Moléculaire de l'Université de Bourgogne, UMR 6302, CNRS, Univ. Bourgogne FrancheComté, 9, Avenue Alain Savary, 21000, Dijon, France; 0000-00022300-4499; E-mail: anthony.romieu@u-bourgogne.fr

\section{Authors}

Garance Dejouy - Institut de Chimie Moléculaire de l'Université de Bourgogne, UMR 6302, CNRS, Univ. Bourgogne FrancheComté, 9, Avenue Alain Savary, 21000, Dijon, France

Quentin Bonnin - Institut de Chimie Moléculaire de l'Université de Bourgogne, UMR 6302, CNRS, Univ. Bourgogne FrancheComté, 9, Avenue Alain Savary, 21000, Dijon, France

Arnaud Chevalier - Institut de Chimie des Substances Naturelles, CNRS UPR 2301, Université Paris-Saclay, 1, Avenue de la Terrasse, 91198 Gif-sur-Yvette, France

Cédric Michaudet - Institut de Chimie Moléculaire de l'Université de Bourgogne, UMR 6302, CNRS, Univ. Bourgogne FrancheComté, 9, Avenue Alain Savary, 21000, Dijon, France

Michel Picquet - Institut de Chimie Moléculaire de l'Université de Bourgogne, UMR 6302, CNRS, Univ. Bourgogne Franche-Comté, 9, Avenue Alain Savary, 21000, Dijon, France

Ibai E. Valverde - Institut de Chimie Moléculaire de l'Université de Bourgogne, UMR 6302, CNRS, Univ. Bourgogne FrancheComté, 9, Avenue Alain Savary, 21000, Dijon, France

\section{Author Contributions}

tThese authors contributed equally to this work.

\section{ACKNOWLEDGMENT}

This work is part of the project "Pharmacoimagerie et Agents Theranostiques", supported by the Universite de Bourgogne and Conseil Régional de Bourgogne through the Plan d'Actions Régional pour l'Innovation (PARI) and the European Union through the PO FEDER-FSE Bourgogne 2014/2020 programs. GDR CNRS "Agents d'Imagerie Moléculaire" (AIM) 2037 is also thanked for its interest in this research topic. G. D. gratefully acknowledges the Burgundy Franche-Comté region for her Ph. D. grant. A further financial support from ANR (AAPG 2018, PRCI LuminoManufacOligo, ANR-18-CE07-0045-01), especially for the post-doc fellowship of K. R., is also greatly acknowledged. COBRA lab (UMR CNRS 6014) and Iris Biotech GmbH are warmly thanked for the generous gift of some (bio)chemical reagents used in this work. The authors also thank the "Plateforme d'Analyse Chimique et de Synthèse Moléculaire de l'Université de Bourgogne" (PACSMUB, http://www.wpcm.fr) for access to spectroscopy instrumentation, Mrs. Marie-José Penouilh (University of Burgundy, PACSMUB) for the recording of HRMS spectra, Dr. Myriam Heydel (University of Burgundy, PACSMUB) for the determination of TFA content in samples purified by semi-preparative RP-HPLC, Dr. Valentin Quesneau (UBFC, ICMUB) for useful discussions and critical reading of the manuscript, and Dr. Laurent Plasseraud (CNRS, ICMUB) for the gift of dimethyl selenide used as reference for ${ }^{77} \mathrm{Se}$ NMR. The Université de Bourgogne, SATT Sayens, Conseil
Régional de Bourgogne Franche-Comté and the french Ministère de l'Enseignement Supérieur et de la Recherche are also gratefully acknowledge for financial support of the analytical platform PACSMUB.

\section{REFERENCES}

(1) (a) Mayer, S. F.; Kroutil, W.; Faber, K. Enzyme-initiated domino (cascade) reactions. Chem. Soc. Rev. 2001, 30, 332-339. (b) Glueck, S. M.; Mayer, S. F.; Kroutil, W.; Faber, K. Advances in biocatalytic synthesis. Enzyme-triggered asymmetric cascade reactions. Pure Appl. Chem. 2002, 74, 2253-2257. (c) Garcia-Junceda, E.; Lavandera, I.; Rother, D.; Schrittwieser, J. H. (Chemo)enzymatic cascades-Nature's synthetic strategy transferred to the laboratory. J. Mol. Catal. B Enzym. 2015, 114, 1-6. (d) Schrittwieser, J. H.; Velikogne, S.; Hall, M.; Kroutil, W. Artificial Biocatalytic Linear Cascades for Preparation of Organic Molecules. Chem. Rev. 2018, 118, 270-348.

(2) (a) Bertozzi, C. R.; Wu, P. In vivo chemistry. Curr. Opin. Chem. Biol. 2013, 17, 717-718. (b) Borrmann, A.; van Hest, J. C. M. Bioorthogonal chemistry in living organisms. Chem. Sci. 2014, 5, 2123-2134. (c) Fox, J. M.; Robillard, M. S. Editorial overview: In vivo chemistry: Pushing the envelope. Curr. Opin. Chem. Biol. 2014, 21, vvii.

(3) (a) Ngo, A. H.; Bose, S.; Do, L. H. Intracellular Chemistry: Integrating Molecular Inorganic Catalysts with Living Systems. Chem. - Eur. J. 2018, 24, 10584-10594. (b) Rebelein, J. G.; Ward, T. R. In vivo catalyzed new-to-nature reactions. Curr. Opin. Biotechnol. 2018 , 53, 106-114.

(4) Yuan, Y.; Liang, G. A biocompatible, highly efficient click reaction and its applications. Org. Biomol. Chem. 2014, 12, 865-871.

(5) Liang, G.; Ren, H.; Rao, J. A biocompatible condensation reaction for controlled assembly of nanostructures in living cells. Nat. Chem. 2010, 2, 54-60.

(6) (a) Ye, D.; Liang, G.; Ma, M. L.; Rao, J. Controlling Intracellular Macrocyclization for the Imaging of Protease Activity. Angew. Chem., Int. Ed. 2011, 50, 2275-2279. (b) Dragulescu-Andrasi, A.; Kothapalli, S.-R.; Tikhomirov, G. A.; Rao, J.; Gambhir, S. S. Activatable Oligomerizable Imaging Agents for Photoacoustic Imaging of FurinLike Activity in Living Subjects. J. Am. Chem. Soc. 2013, 135, 11015 11022. (c) Godinat, A.; Park, H. M.; Miller, S. C.; Cheng, K.; Hanahan, D.; Sanman, L. E.; Bogyo, M.; Yu, A.; Nikitin, G. F.; Stahl, A.; Dubikovskaya, E. A. A Biocompatible in Vivo Ligation Reaction and Its Application for Noninvasive Bioluminescent Imaging of Protease Activity in Living Mice. ACS Chem. Biol. 2013, 8, 987-999. (d) Shen, B.; Jeon, J.; Palner, M.; Ye, D.; Shuhendler, A.; Chin, F. T.; Rao, J. Positron Emission Tomography Imaging of Drug-Induced Tumor Apoptosis with a Caspase-Triggered Nanoaggregation Probe. Angew. Chem., Int. Ed. 2013, 52, 10511-10514. (e) Van de Bittner, G. C.; Bertozzi, C. R.; Chang, C. J. Strategy for Dual-Analyte Luciferin Imaging: In Vivo Bioluminescence Detection of Hydrogen Peroxide and Caspase Activity in Murine Model of Acute Inflammation. J. Am. Chem. Soc. 2013, 135, 1783-1795. (f) Ye, D.; Pandit, P.; Kempen, P.; Lin, J.; Xiong, L.; Sinclair, R.; Rutt, B.; Rao, J. Redox-Triggered SelfAssembly of Gadolinium-Based MRI Probes for Sensing Reducing Environment. Bioconjugate Chem. 2014, 25, 1526-1536. (g) Ye, D.; Shuhendler, A. J.; Cui, L.; Tong, L.; Tee, S. S.; Tikhomirov, G.; Felsher, D. W.; Rao, J. Bioorthogonal cyclization-mediated in situ selfassembly of small-molecule probes for imaging caspase activity in vivo. Nat. Chem. 2014, 6, 519-526. (h) Ye, D.; Shuhendler, A. J.; Pandit, P.; Brewer, K. D.; Tee, S. S.; Cui, L.; Tikhomirov, G.; Rutt, B.; Rao, J. Caspase-responsive smart gadolinium-based contrast agent for magnetic resonance imaging of drug-induced apoptosis. Chem. Sci. 2014, 5, 3845-3852. (i) Nejadnik, H.; Ye, D.; Lenkov, O. D.; Donig, J. S.; Martin, J. E.; Castillo, R.; Derugin, N.; Sennino, B.; Rao, J.; Daldrup-Link, H. Magnetic Resonance Imaging of Stem Cell Apoptosis in Arthritic Joints with a Caspase Activatable Contrast Agent. ACS Nano 2015, 9, 1150-1160. (j) Yuan, Y.; Zhang, J.; Qi, X.; Li, S.; Liu, G.; Siddhanta, S.; Barman, I.; Song, X.; McMahon, M. T.; Bulte, J. W. M. Furin-mediated intracellular self-assembly of 
olsalazine nanoparticles for enhanced magnetic resonance imaging and tumour therapy. Nat. Mater. 2019, 18, 1376-1383. (k) Chen, Z.; Chen, M.; Cheng, Y.; Kowada, T.; Xie, J.; Zheng, X.; Rao, J. Exploring the Condensation Reaction between Aromatic Nitriles and Amino Thiols To Optimize In Situ Nanoparticle Formation for the Imaging of Proteases and Glycosidases in Cells. Angew. Chem., Int. Ed. 2020, 59, 3272-3279. (1) Chen, Z.; Chen, M.; Zhou, K.; Rao, J. Pre-targeted Imaging of Protease Activity through In Situ Assembly of Nanoparticles. Angew. Chem., Int. Ed. 2020, 59, 7864-7870.

(7) Kim, T.-H.; Swager, T. M. A Fluorescent Self-Amplifying Wavelength-Responsive Sensory Polymer for Fluoride Ions. Angew. Chem. Int. Ed. 2003, 42, 4803-4806.

(8) For reviews, see: (a) Anslyn, E. V. Supramolecular and Chemical Cascade Approaches to Molecular Sensing. J. Am. Chem. Soc. 2010, 132, 15833-15835. (b) Luo, X.; Gu, L.; Qian, X. H.; Yang, Y. C. Molecular Probe Design via the "Covalent-Assembly" Principle. Chem. Commun. 2020, in press, DOI: 10.1039/D0CC00542H.

(9) Yang, Y.; Seidlits, S. K.; Adams, M. M.; Lynch, V. M.; Schmidt, C. E.; Anslyn, E. V.; Shear, J. B. A Highly Selective Low-Background Fluorescent Imaging Agent for Nitric Oxide. J. Am. Chem. Soc. 2010, $132,13114-13116$

(10) Romieu, A. "AND" luminescent "reactive" molecular logic gates: a gateway to multi-analyte bioimaging and biosensing. Org. Biomol. Chem. 2015, 13, 1294-1306.

(11) (a) Kim, T.-I.; Jeong, M. S.; Chung, S. J.; Kim, Y. An Iminocoumarin-Based Fluorescent Probe for the Selective Detection of Dual-Specific Protein Tyrosine Phosphatases. Chem. - Eur. J. 2010, 16, 5297-5300. (b) Kim, T.-I.; Kim, H.; Choi, Y.; Kim, Y. A fluorescent turn-on probe for the detection of alkaline phosphatase activity in living cells. Chem. Commun. 2011, 47, 9825-9827. (c) Kim, D.; Sambasivan, S.; Nam, H.; Hean Kim, K.; Yong Kim, J.; Joo, T.; Lee, K.-H.; Kim, K.-T.; Han Ahn, K. Reaction-based two-photon probes for in vitro analysis and cellular imaging of monoamine oxidase activity. Chem. Commun. 2012, 48, 6833-6835. (d) Pershagen, E.; Nordholm, J.; Borbas, K. E. Luminescent Lanthanide Complexes with AnalyteTriggered Antenna Formation. J. Am. Chem. Soc. 2012, 134, 98329835. (e) Park, J.; Kim, Y. An improved fluorogenic substrate for the detection of alkaline phosphatase activity. Bioorg. Med. Chem. Lett. 2013, 23, 2332-2335. (f) Debieu, S.; Romieu, A. Dual enzymeresponsive "turn-on" fluorescence sensing systems based on in situ formation of 7-hydroxy-2-iminocoumarin scaffolds. Org. Biomol. Chem. 2015, 13, 10348-10361. (g) Pershagen, E.; Borbas, K. E. Multiplex Detection of Enzymatic Activity with Responsive Lanthanide-Based Luminescent Probes. Angew. Chem., Int. Ed. 2015, 54, 1787-1790. (h) Xia, L.; Hu, F.; Huang, J.; Li, N.; Gu, Y.; Wang, P. A fluorescent turn-on probe for nitroreductase imaging in living cells and tissues under hypoxia conditions. Sens. Actuator B-Chem. 2018, 268, 70-76. (i) Kong, F.; Li, Y.; Yang, C.; Li, X.; Wu, J.; Liu, X.; Gao, X.; Xu, K.; Tang, B. A fluorescent probe for simultaneously sensing NTR and hNQO1 and distinguishing cancer cells. J. Mater. Chem. B 2019, 7, 6822-6827. (j) Liu, Y.; Yan, W.; Li, H.; Peng, H.; Suo, X.; Li, Z.; Liu, H.; Zhang, J.; Wang, S.; Liu, D. Two-photon fluorescent probe for hypoxic cancer stem cells by responding to endogenous nitroreductase. Anal. Methods 2019, 11, 421-426. (k) Qin, H.; Li, L.; $\mathrm{Li}, \mathrm{K}$; Xiaoqi, Y. Novel strategy of constructing fluorescent probe for MAO-B via cascade reaction and its application in imaging MAO-B in human astrocyte. Chin. Chem. Lett. 2019, 30, 71-74.

(12) For the sole examples of fluorogenic prodrugs based on the "covalent-assembly" probe design principle, see: (a) Feng, W.; Gao, C.; Liu, W.; Ren, H.; Wang, C.; Ge, K.; Li, S.; Zhou, G.; Li, H.; Wang, S.; Jia, G.; Li, Z.; Zhang, J. A novel anticancer theranostic pro-prodrug based on hypoxia and photo sequential control. Chem. Commun. 2016, 52, 9434-9437. (b) Liu, W.; Liu, H.; Peng, X.; Zhou, G.; Liu, D.; Li, S.; Zhang, J.; Wang, S. Hypoxia-Activated Anticancer Prodrug for Bioimaging, Tracking Drug Release, and Anticancer Application. Bioconjugate Chem. 2018, 29, 3332-3343. (c) Peng, X.; Gao, J.; Yuan, Y.; Liu, H.; Lei, W.; Li, S.; Zhang, J.; Wang, S. Hypoxia-Activated and
Indomethacin-Mediated Theranostic Prodrug Releasing Drug OnDemand for Tumor Imaging and Therapy. Bioconjugate Chem. 2019, 30, 2828-2843.

(13) (a) Lei, Z.; Yang, Y. A Concise Colorimetric and Fluorimetric Probe for Sarin Related Threats Designed via the "CovalentAssembly" Approach. J. Am. Chem. Soc. 2014, 136, 6594-6597. (b) Song, L.; Lei, Z.; Zhang, B.; Xu, Z.; Li, Z.; Yang, Y. A zerobackground fluorescent probe for $\mathrm{Hg}^{2+}$ designed via the "covalentassembly" principle. Anal. Methods 2014, 6, 7597-7600. (c) Lei, Z.; Zeng, Z.; Qian, X.; Yang, Y. A novel chromogenic and fluorogenic scaffold for detection of oxidative radicals. Chin. Chem. Lett. 2017, 28, 2001-2004

(14) (a) Debieu, S.; Romieu, A. In situ formation of pyronin dyes for fluorescence protease sensing. Org. Biomol. Chem. 2017, 15, 25752584. (b) Debieu, S.; Romieu, A. Kinetics improvement of proteasemediated formation of pyronin dyes. Tetrahedron Lett. 2018, 59, 19401944. (c) Renault, K.; Debieu, S.; Richard, J.-A.; Romieu, A. Deeper insight into the protease-sensitive "covalent-assembly" fluorescent probes for practical biosensing applications. Org. Biomol. Chem. 2019, 17, 8918-8932.

(15) Wang, J.; Li, C.; Chen, Q.; Li, H.; Zhou, L.; Jiang, X.; Shi, M.; Zhang, P.; Jiang, G.; Tang, B. Z. An Easily Available Ratiometric Reaction-Based AIE Probe for Carbon Monoxide Light-up Imaging. Anal. Chem. 2019, 91, 9388-9392.

(16) Carta, A.; Piras, S.; Loriga, G.; Paglietti, G. Chemistry, biological properties, and SAR analysis of quinoxalinones. Mini-Rev. Med. Chem. 2006, 6, 1179-1200.

(17) (a) Hara, S.; Yamaguchi, M.; Nakamura, M.; Ohkura, Y. Fluorescent products of reaction between $\alpha$-keto acids and 1,2diamino-4,5-dimethoxybenzene. Chem. Pharm. Bull. 1985, 33, 3493 8. (b) Renault, K.; Renard, P.-Y.; Sabot, C. Photophysical properties of quinoxalin-2(1H)-ones: application in the preparation of an azidebased fluorogenic probe for the detection of hydrogen sulfide. New $J$. Chem. 2017, 41, 10432-10437.

(18) Shi, L.; Zhou, H.; Wu, J.; Li, X. Advances in the chemistry of quinoxalinone derivatives. Mini-Rev. Org. Chem. 2015, 12, 96-112.

(19) Chandel, A. K.; Rao, L. V.; Narasu, M. L.; Singh, O. V. The realm of penicillin $\mathrm{G}$ acylase in $\beta$-lactam antibiotics. Enzyme Microb. Technol. 2008, 42, 199-207.

(20) Pessah, N.; Reznik, M.; Shamis, M.; Yantiri, F.; Xin, H.; Bowdish, K.; Shomron, N.; Ast, G.; Shabat, D. Bioactivation of carbamate-based 20(S)-camptothecin prodrugs. Bioorg. Med. Chem. 2004, 12, 1859-1866.

(21) Ottenwaelder, X.; Ruiz-Garcia, R.; Blondin, G.; Carasco, R.; Cano, J.; Lexa, D.; Journaux, Y.; Aukauloo, A. From metal to ligand electroactivity in nickel(II) oxamato complexes. Chem. Commun. 2004, 504-505.

(22) For selected reviews, see: (a) Alouane, A.; Labruère, R.; Le Saux, T.; Schmidt, F.; Jullien, L. Self-Immolative Spacers: Kinetic Aspects, Structure-Property Relationships, and Applications. Angew. Chem., Int. Ed. 2015, 54, 7492-7509. (b) Yan, J.; Lee, S.; Zhang, A.; Yoon, J. Self-immolative colorimetric, fluorescent and chemiluminescent chemosensors. Chem. Soc. Rev. 2018, 47, 69006916.

(23) Detty, M. R.; Prasad, P. N.; Donnelly, D. J.; Ohulchanskyy, T.; Gibson, S. L.; Hilf, R. Synthesis, properties, and photodynamic properties in vitro of heavy-chalcogen analogues of tetramethylrosamine. Bioorg. Med. Chem. 2004, 12, 2537-2544.

(24) Mendive-Tapia, L.; Zhao, C.; Akram, A. R.; Preciado, S.; Albericio, F.; Lee, M.; Serrels, A.; Kielland, N.; Read, N. D.; Lavilla, R.; Vendrell, M. Spacer-free BODIPY fluorogens in antimicrobial peptides for direct imaging of fungal infection in human tissue. Nat. Commun. 2016, 7, 10940, DOI: 10.1038/ncomms10940.

(25) Gomes, P.; Vale, N.; Moreira, R. Cyclization-activated Prodrugs. Molecules 2007, 12, 2484-2506. 